\title{
P02.172. 1-year sustaining efficacy of multidimensional therapy for inpatients with different conditions of chronic musculoskeletal pain
}

\author{
R Stange ${ }^{1 *}$, U Hackermeier ${ }^{1}$, G Franzen ${ }^{1}$, T Ostermann $^{2}$, B Uehleke $^{1}$, A Michalsen $^{1}$ \\ From International Research Congress on Integrative Medicine and Health 2012 \\ Portland, Oregon, USA. 15-18 May 2012
}

\section{Purpose}

Chronic pain is the predominant condition for use of complementary medicine in Western countries. While many modalities have proven efficacy as a single intervention, therapeutical failure is often due to longer histories, comorbidities or complex etiologic conditions. Therefore, individually tailored therapies may show more efficacy, but little research could be retrieved. We investigated outcomes of inpatients after an intensive period of approximatey 2 weeks with individual combinations of therapies, composed of diet and fasting, physical therapy, relaxation, herbs, acupuncture, and neural therapy.

\section{Methods}

Ongoing inpatients (age $18-70, \mathrm{M}$ and F) with chronic musculoskeletal pain of different origin (Gerbershagen classification III and IV, multi-morbidity allowed) for more than 2 years were included. Main outcome parameters were changes in VAS (0-100) for global pain and SF-36 between T1 before therapy and final visit T5 after 1 year with no further protocol treatment. Results of this pivotal uncontrolled trial were interpreted as descriptive.

\section{Results}

Two hundred twenty-one patients (intent to treat, mean age $57.2+12.2 \mathrm{y}$ ) were enrolled with full data sets from 101 (per protocol). Most frequent diagnoses were low back pain $(\mathrm{n}=53,24.0 \%)$, fibromyalgia $(44,19.9 \%)$, rheumatoid arthritis $(25,11.3 \%)$, and chronic neck pain (22, $10.0 \%)$. Mean VAS decreased by 15.1 from $60.7+23.0$
(T1) to $45.6+26.2$ (T5) ( $<<0.0001$, two-sided t-test), with highest improvement for low back pain (decrease of 17.5) and no differentiation for multi-morbidity ( $\mathrm{n}=46$ with, $\mathrm{n}=55$ without). SF-36 physical and mental component scores improved significantly from $40.0+12.2$ to 44.3 +12.5 and from $29.6+8.2$ to $32.9+10.5$, respectively $(\mathrm{p}<0.0001$ for each).

\section{Conclusion}

An individual multidimensional treatment for chronic musculoskeletal pain with inpatients may result in sustaining beneficial effects over one year. Surprisingly, differential improvement in quality of life extended to both physical and emotional dimensions. Non-compliance was attributed to the long interval between the end of therapy and visit for primary outcome measurement.

\section{Author details \\ ${ }^{1}$ Charité, University of Medicine and Immanuel Hospital, Berlin, Germany. ${ }^{2}$ Center of Integrative Medicine, Dprtmt. of Medicine, Witten, Germany.}

Published: 12 June 2012

doi:10.1186/1472-6882-12-S1-P228

Cite this article as: Stange et al:: P02.172. 1-year sustaining efficacy of multidimensional therapy for inpatients with different conditions of chronic musculoskeletal pain. BMC Complementary and Alternative Medicine 2012 12(Suppl 1):P228. 